Pure and Applied Mathematics Quarterly

Volume 5, Number 4

(Special Issue: In honor of

John Tate, Part 1 of 2)

1201-1217, 2009

\title{
Canonical Heights and the Arithmetic Complexity of Morphisms on Projective Space
}

\author{
Shu Kawaguchi and Joseph H. Silverman
}

For John Tate on the occasion of his $80^{\text {th }}$ birthday.

\begin{abstract}
Let $\varphi, \psi: \mathbb{P}^{N} \rightarrow \mathbb{P}^{N}$ be morphisms of degree $d \geq 2$ defined over $\overline{\mathbb{Q}}$. We define the arithmetic distance between $\varphi$ and $\psi$ to be the supremum of the difference of their canonical heights,

$$
\hat{\delta}(\varphi, \psi)=\sup _{P \in \mathbb{P}^{N}(\overline{\mathbb{Q}})}\left|\hat{h}_{\varphi}(P)-\hat{h}_{\psi}(P)\right| .
$$

We prove comparison theorems relating $\hat{\delta}(\varphi, \psi)$ to other height functions and we show that for a fixed $\psi$, the set of $\varphi$ satisfying $\hat{\delta}(\varphi, \psi) \leq B$ and $\operatorname{deg}(\varphi)=d$ is a set of bounded height. In particular, there are only finitely many such $\varphi$ defined over any given number field.
\end{abstract}

Keywords: canonical height, arithmetic complexity, arithmetic of dynamical systems

Received June 27, 2006.

1991 Mathematics Subject Classification. Primary: 11G50; Secondary: 14G40, 37F10.

The first author's research supported by grant-in-aid for young scientists (B) 18740008 . He thanks the Institut de Mathématiques de Jussieu and Vincent Maillot for their warm hospitality. The second author's research supported by NSA grant H98230-04-1-0064. 


\section{PRELUde}

The theory of canonical heights on abelian varieties originated with the work of Néron [10] and Tate (first described in print by Manin [8]) in 1965. Tate's simple and elegant limit construction uses a Cauchy sequence telescoping sum argument. Néron's construction, which is via more delicate local tools, has proven to be fundamental for understanding the deeper properties of the canonical height.

Canonical heights appear prominently in the conjecture of Birch and Swinnerton-Dyer, so early efforts to check the conjecture numerically required the computation of $\hat{h}(P)$ to at least a few decimal places. In the mid-1970's, John Coates used Tate's limit definition/construction to compute $\hat{h}(P)$ to three decimal places and he mentioned the computation during a talk at the Harvard Number Theory Seminar. That evening at a party in Coates' honor, Tate pulled out a primitive Texas Instruments programmable calculator, punched in a few values, and in a fraction of a second recomputed Coates' value of $\hat{h}(P)$ to 8 decimal places! The method was via a rapidly converging infinite series for Néron's local canonical heights that Tate had described in an (unpublished) letter to Serre. Tate generously shared copies of his letter with other mathematicians, including the second author of this paper (who was at the time a mere graduate student), and Tate's numerically efficient series for the computation of canonical heights appeared in [13] and, in generalized form, in [11].

The importance of canonical heights in arithmetic geometry and related fields has continued to grow, for example in arithmetic intersection theory, Arakelov geometry, special values of $L$-functions, cryptography, and dynamical systems. The present paper is devoted to an application of the theory of canonical heights to study the arithmetic properties of dynamical systems.

\section{INTRODUCTION}

Let

$$
h: \mathbb{P}^{N}(\overline{\mathbb{Q}}) \longrightarrow \mathbb{R}
$$

be the standard (absolute logarithmic) Weil height $[4, \S \mathrm{B} .2]$ and let

$$
\varphi: \mathbb{P}^{N} \longrightarrow \mathbb{P}^{N}
$$


be a morphism of degree $d \geq 2$ defined over $\overline{\mathbb{Q}}$. Associated to $\varphi$ is a canonical height function

$$
\hat{h}_{\varphi}: \mathbb{P}^{N}(\overline{\mathbb{Q}}) \longrightarrow \mathbb{R}
$$

defined via the Tate limit

$$
\hat{h}_{\varphi}(P)=\lim _{n \rightarrow \infty} \frac{1}{d^{n}} h\left(\varphi^{n}(P)\right)
$$

and having the agreeable properties

$$
\hat{h}_{\varphi}=h+O(1) \quad \text { and } \quad \hat{h}_{\varphi} \circ \varphi=d \hat{h}_{\varphi} .
$$

(See $[4, \S$ B.4] for details.) In an earlier paper [6] the authors considered under what circumstances two morphisms $\varphi$ and $\psi$ can have identical canonical heights $\hat{h}_{\varphi}=\hat{h}_{\psi}$. In this note we take up the question of the extent to which the difference $\hat{h}_{\varphi}-\hat{h}_{\psi}$ is an intrinsic measure of the arithmetic distance between the maps $\varphi$ and $\psi$.

More precisely, we define the arithmetic distance between two morphisms $\varphi, \psi$ : $\mathbb{P}^{N} \rightarrow \mathbb{P}^{N}$ to be the quantity

$$
\hat{\delta}(\varphi, \psi)=\sup _{P \in \mathbb{P}^{N}(\overline{\mathbb{Q}})}\left|\hat{h}_{\varphi}(P)-\hat{h}_{\psi}(P)\right| .
$$

Note that $\hat{\delta}(\varphi, \psi)$ is finite, since $\hat{h}_{\varphi}=h+O(1)$ and $\hat{h}_{\psi}=h+O(1)$. Further, $\hat{\delta}(\varphi, \psi)=0$ if and only if $\hat{h}_{\varphi}=\hat{h}_{\psi}$, and an elementary application of the triangle inequality (Lemma 5 ) yields

$$
\hat{\delta}(\varphi, \psi) \leq \hat{\delta}(\varphi, \lambda)+\hat{\delta}(\lambda, \psi) .
$$

The principal result in this note is a comparison theorem showing that the arithmetic distance between $\varphi$ and $\psi$ is related to the naive height of $\varphi$ and $\psi$ as defined by the coefficients of their defining polynomials.

More intrinsically, the set of morphisms $\mathbb{P}^{N} \rightarrow \mathbb{P}^{N}$ of degree $d$, which we denote by $\operatorname{Mor}_{d}^{N}$, is naturally identified with a Zariski open subset of a projective space via

$$
\operatorname{Mor}_{d}^{N} \subset \mathbb{P}^{L}, \quad \varphi=\left[\varphi_{0}: \cdots: \varphi_{N}\right] \longmapsto\left[\text { coefficients of } \varphi_{0}, \ldots, \varphi_{N}\right] .
$$

Defining $h(\varphi)$, the Weil height of $\varphi$, to be the height of the corresponding point in $\mathbb{P}^{L}$, we prove the following result. 
Theorem 1. Let $\varphi, \psi: \mathbb{P}^{N} \rightarrow \mathbb{P}^{N}$ be morphisms of degree at least 2 defined over $\overline{\mathbb{Q}}$. Then

$$
\hat{\delta}(\varphi, \psi)-h(\psi) \ll h(\varphi) \ll \hat{\delta}(\varphi, \psi)+h(\psi),
$$

where the implied constants depend only on $N$ and the degrees of $\varphi$ and $\psi$. (See Theorem 15 for an explicit upper bound.)

An immediate corollary of Theorem 1 and Northcott's theorem [4, B.2.3] is the following finiteness theorem.

Corollary 2. Fix a morphism $\psi: \mathbb{P}^{N} \rightarrow \mathbb{P}^{N}$ of degree at least 2 defined over $\overline{\mathbb{Q}}$ and an integer $d \geq 2$. Then for all $B>0$ the set of morphisms $\varphi \in \operatorname{Mor}_{d}^{N}(\overline{\mathbb{Q}})$ satisfying

$$
\hat{\delta}(\varphi, \psi) \leq B
$$

is a set of bounded height in $\operatorname{Mor}_{d}^{N}(\overline{\mathbb{Q}}) \subset \mathbb{P}^{L}(\overline{\mathbb{Q}})$. In particular, there are only finitely many such $\varphi$ defined over number fields of bounded degree over $\mathbb{Q}$.

The proof of Theorem 1 involves a number of steps. It turns out to be more convenient to consider another sort of arithmetic distance function defined by

$$
\hat{\Delta}_{\psi}(\varphi)=\sup _{P \in \mathbb{P}^{N}(\overline{\mathbb{Q}})}\left|\frac{1}{\operatorname{deg}(\varphi)} \hat{h}_{\psi}(\varphi(P))-\hat{h}_{\psi}(P)\right| .
$$

An elementary argument relates $\hat{\Delta}_{\psi}(\varphi)$ to $\hat{\delta}(\varphi, \psi)$. The triangle inequality for $\hat{\delta}$ allows us to reduce to the case that $\psi$ is the powering map, so $\hat{h}_{\psi}$ is the Weil height $h$, in which case we write simply $\hat{\Delta}(\varphi)$. Finally, and this is the heart of the argument, we prove a theorem comparing $\hat{\Delta}(\varphi)$ to $h(\varphi)$. For the lower bound we consider the universal family of morphisms $\mathbb{P}^{N} \rightarrow \mathbb{P}^{N}$ over $\operatorname{Mor}_{d}^{N}$ and apply general results of Call and Silverman [3]. For the upper bound we use a matrix calculation to prove an explicit inequality

$$
h(\varphi) \leq d\left(\begin{array}{c}
N+d \\
N
\end{array}\right) \hat{\Delta}(\varphi)+O_{N, d}(1) .
$$

\section{Arithmetic Complexity}

The results proven in [6] show that morphisms with identical canonical heights are very closely related to one another. This suggests using the difference between canonical heights as a way to measure the arithmetic distance between the morphisms, which leads us to make the following definitions. 
Definition. Let $\varphi, \psi: \mathbb{P}^{N} \rightarrow \mathbb{P}^{N}$ be morphisms defined over $\overline{\mathbb{Q}}$ of degree at least 2 . We use the canonical height to define two arithmetic distance functions,

$$
\begin{aligned}
& \hat{\delta}(\varphi, \psi)=\sup _{P \in \mathbb{P}^{N}(\overline{\mathbb{Q}})}\left|\hat{h}_{\varphi}(P)-\hat{h}_{\psi}(P)\right|, \\
& \hat{\Delta}_{\psi}(\varphi)=\sup _{P \in \mathbb{P}^{N}(\overline{\mathbb{Q}})}\left|\frac{1}{\operatorname{deg}(\varphi)} \hat{h}_{\psi}(\varphi(P))-\hat{h}_{\psi}(P)\right| .
\end{aligned}
$$

In particular, $\hat{\delta}(\varphi, \psi)=0$ if and only if $\hat{h}_{\varphi}=\hat{h}_{\psi}$.

In the special case that $\psi$ is the power map

$$
\left[x_{0}, \ldots, x_{N}\right] \longmapsto\left[x_{0}^{d}, \ldots, x_{N}^{d}\right]
$$

so $\hat{h}_{\psi}$ is the usual Weil height $h$, we write simply $\hat{\delta}(\varphi)$ and $\hat{\Delta}(\varphi)$. We call $\hat{\delta}(\varphi)$ the arithmetic complexity of $\varphi$.

Remark 3 . The canonical heights $\hat{h}_{\varphi}$ and $\hat{h}_{\psi}$ satisfy

$$
\hat{h}_{\varphi}=h+O(1) \quad \text { and } \quad \hat{h}_{\psi}=h+O(1)
$$

and the Weil height $h$ satisfies $h(\varphi(P))=\operatorname{deg}(\varphi) h(P)+O(1)$, so the suprema used to define $\hat{\delta}(\varphi, \psi)$ and $\hat{\Delta}_{\psi}(\varphi)$ are finite.

Remark 4. The canonical height, and a fortiori the arithmetic distance $\hat{\delta}(\varphi, \psi)$, are only defined for maps of degree at least 2 . However, we observe that $\hat{\Delta}_{\psi}(\varphi)$ is well-defined also for $\operatorname{deg}(\varphi)=1$.

We begin with an elementary triangle inequality for $\hat{\delta}$.

Lemma 5. Let $\varphi, \psi, \nu: \mathbb{P}^{N} \rightarrow \mathbb{P}^{N}$ be morphisms defined over $\overline{\mathbb{Q}}$ of degree at least 2. Then

$$
\hat{\delta}(\varphi, \psi) \leq \hat{\delta}(\varphi, \nu)+\hat{\delta}(\nu, \psi)
$$

Proof. This is immediate by taking suprema of

$$
\left|\hat{h}_{\varphi}(P)-\hat{h}_{\psi}(P)\right| \leq\left|\hat{h}_{\varphi}(P)-\hat{h}_{\nu}(P)\right|+\left|\hat{h}_{\nu}(P)-\hat{h}_{\psi}(P)\right| .
$$

We next prove a comparison theorem for $\hat{\delta}$ and $\hat{\Delta}$. 
Proposition 6. Let $\varphi, \psi: \mathbb{P}^{N} \rightarrow \mathbb{P}^{N}$ be morphisms defined over $\overline{\mathbb{Q}}$ of degree at least 2 and let $d_{\varphi}=\operatorname{deg}(\varphi)$. Then

$$
\frac{d_{\varphi}}{d_{\varphi}+1} \hat{\Delta}_{\psi}(\varphi) \leq \hat{\delta}(\varphi, \psi) \leq \frac{d_{\varphi}}{d_{\varphi}-1} \hat{\Delta}_{\psi}(\varphi)
$$

Proof. We compute directly using the definitions of $\hat{\delta}$ and $\hat{\Delta}$, the triangle inequality, and basic properties of the canonical height.

$$
\begin{aligned}
& \hat{\Delta}_{\psi}(\varphi)=\sup _{P \in \mathbb{P}^{N}(\overline{\mathbb{Q}})}\left|\frac{1}{d_{\varphi}} \hat{h}_{\psi}(\varphi(P))-\hat{h}_{\psi}(P)\right| \\
& \quad \leq \sup _{P \in \mathbb{P}^{N}(\overline{\mathbb{Q}})} \frac{1}{d_{\varphi}}\left|\hat{h}_{\psi}(\varphi(P))-\hat{h}_{\varphi}(\varphi(P))\right|+\sup _{P \in \mathbb{P}^{N}(\overline{\mathbb{Q}})}\left|\hat{h}_{\varphi}(P)-\hat{h}_{\psi}(P)\right| \\
& \quad=\frac{1}{d_{\varphi}} \hat{\delta}(\varphi, \psi)+\hat{\delta}(\varphi, \psi) .
\end{aligned}
$$

This gives one inequality. The other is proven similarly. Thus

$$
\begin{aligned}
& \hat{\Delta}_{\psi}(\varphi)=\sup _{P \in \mathbb{P}^{N}(\overline{\mathbb{Q}})}\left|\frac{1}{d_{\varphi}} \hat{h}_{\psi}(\varphi(P))-\hat{h}_{\psi}(P)\right| \\
& \quad \geq \sup _{P \in \mathbb{P}^{N}(\overline{\mathbb{Q}})}\left|\hat{h}_{\psi}(P)-\hat{h}_{\varphi}(P)\right|-\sup _{P \in \mathbb{P}^{N}(\overline{\mathbb{Q}})} \frac{1}{d_{\varphi}}\left|\hat{h}_{\varphi}(\varphi(P))-\hat{h}_{\psi}(\varphi(P))\right| \\
& \quad=\hat{\delta}(\varphi, \psi)-\frac{1}{d_{\varphi}} \hat{\delta}(\varphi, \psi) .
\end{aligned}
$$

This completes the proof of the proposition.

Remark 7. Let $\varphi=\left(\varphi_{1}, \varphi_{2}, \ldots\right)$ be a sequence of morphisms $\varphi_{i}: \mathbb{P}^{N} \rightarrow \mathbb{P}^{N}$ of degree $\operatorname{deg}\left(\varphi_{i}\right) \geq 2$. The arithmetic complexity of the sequence $\varphi$ is the quantity

$$
\hat{\delta}(\varphi)=\sup _{i \geq 1} \hat{\delta}\left(\varphi_{i}\right)
$$

and we say that the sequence $\boldsymbol{\varphi}$ is (arithmetically) bounded if $\hat{\delta}(\boldsymbol{\varphi})$ is finite. It is shown in [5] that there is a canonical height function $\hat{h}_{\varphi}$ naturally associated to every arithmetically bounded sequence. (For a canonical height function associated to a sequence that is not necessarily arithmetically bounded, see $[5$, Remark 3.8].) A consequence of Theorem 16 proven below is that if $\varphi$ is arithmetically bounded and contains infinitely many distinct maps, then either the degrees $\operatorname{deg}\left(\varphi_{i}\right)$ of the maps or the degrees of the fields of definition $\mathbb{Q}\left(\varphi_{i}\right)$ must go to infinity. 


\section{A COMPARISON THEOREM FOR $h(\varphi)$ AND $\hat{\Delta}(\varphi)$}

The arithmetic complexity $\hat{\delta}(\varphi)$ of a rational map is an intrinsic measure of the extent to which $\varphi$ differs arithmetically from the elementary power map (2). A more naive way to measure the arithmetic complexity of $\varphi$ is to take the height of its coefficients. In this section we relate these two notions. This will be used in the next section to show that, in a suitable sense, there are only finitely many rational maps of bounded complexity.

We write $\operatorname{Rat}_{d}^{N}$ for the set of rational maps $\varphi: \mathbb{P}^{N} \rightarrow \mathbb{P}^{N}$ of degree $d$. This set is naturally identified with a subset of a projective space $\mathbb{P}^{L}$ by writing $\varphi=\left[\varphi_{0}: \cdots: \varphi_{N}\right]$ and using the coefficients of the homogeneous polynomials $\varphi_{0}, \ldots, \varphi_{N}$ as homogeneous coordinates in $\mathbb{P}^{L}$. The subset $\operatorname{Mor}_{d}^{N}$ of $\operatorname{Rat}_{d}^{N}$ consisting of morphisms is an affine subset of $\mathbb{P}^{L}$. (In fact, it is the complement of the hypersurface defined by the Macaulay resultant of $\varphi_{0}, \ldots, \varphi_{N}$.)

Definition. Let $\varphi: \mathbb{P}^{N} \rightarrow \mathbb{P}^{N}$ be a rational map of degree $d$ defined over $\overline{\mathbb{Q}}$. We define the Weil height of $\varphi$ to be the height of the corresponding point in $\operatorname{Rat}_{d}^{N}(\overline{\mathbb{Q}}) \subset \mathbb{P}^{L}(\overline{\mathbb{Q}})$. We denote this height by $h(\varphi)$. Similarly, the height $h(F)$ of a nonzero homogeneous polynomial $F \in \overline{\mathbb{Q}}\left[x_{0}, \ldots, x_{N}\right]$ is the height of the point in projective space defined by its coordinates.

Theorem 8. Let $N \geq 1$ and $d \geq 1$ be given. There are constants $c_{1}, c_{2}, c_{3}>0$, depending only on $N$ and $d$, so that for all morphisms $\varphi: \mathbb{P}^{N} \rightarrow \mathbb{P}^{N}$ of degree $d \geq 1$ defined over $\overline{\mathbb{Q}}$,

$$
c_{1} \hat{\Delta}(\varphi)-c_{2} \leq h(\varphi) \leq d\left(\begin{array}{c}
N+d \\
N
\end{array}\right) \hat{\Delta}(\varphi)+c_{3} .
$$

Remark 9. We give an example with $N=1$ that illustrates the upper bound in the theorem. Let $\varphi_{A}(x)=x^{d}+A x^{d-1}$ with $A \in \mathbb{Z}, A \neq 0$. Then one easily checks that for all $\alpha \in \overline{\mathbb{Q}}$,

$$
\left|\frac{1}{d} h\left(\varphi_{A}(\alpha)\right)-h(\alpha)\right| \leq \frac{1}{d} \log (1+|A|)=\frac{1}{d} h\left(\varphi_{A}\right)+O(1 /|A|) .
$$

Taking the supremum over $\alpha$ yields $d \hat{\Delta}\left(\varphi_{A}\right) \leq h\left(\varphi_{A}\right)+O(1 /|A|)$, and hence

$$
\limsup _{|A| \rightarrow \infty} \frac{h\left(\varphi_{A}\right)}{\hat{\Delta}\left(\varphi_{A}\right)} \geq d
$$

This may be compared with the upper bound of $d^{2}+d$ provided by the theorem. 
Remark 10. In general, we consider the limit

$$
\alpha(N, d)=\limsup _{\substack{\varphi \in \operatorname{Mor}_{d}^{N}(\overline{\mathbb{Q}}) \\
h(\varphi) \rightarrow \infty}} \frac{h(\varphi)}{\hat{\Delta}(\varphi)} \leq d\left(\begin{array}{c}
N+d \\
N
\end{array}\right),
$$

where the upper bound is provided by Theorem 8. It would be interesting to improve this upper bound and/or to obtain nontrivial lower bounds for $\alpha(N, d)$.

Proof of the Lower Bound in Theorem 8. We prove the lower bound in (3) by showing that it is a special case of [3, Theorem 3.1]. In the notation of [3], we take $T^{0}$ to be the set of morphisms $\mathbb{P}^{N} \rightarrow \mathbb{P}^{N}$ of degree $d$. Thus $T^{0}$ is naturally an open subset of $\mathbb{P}^{L}$ and we set $T=\mathbb{P}^{L}$. Then we let $\mathcal{V}=\mathbb{P}^{N} \times T$, we let $\mathcal{V} \rightarrow T$ be projection onto the second factor, and we let $\varphi: \mathcal{V} \rightarrow \mathcal{V}$ be the rational map whose restriction to the generic fiber is the generic degree $d$ morphism from $\mathbb{P}^{N}$ to itself. We further let $\eta$ be a divisor class in $\operatorname{Pic}(\mathcal{V})$ whose restriction to the generic fiber is a hyperplane section. Then [3, Theorem 3.1] says that there are (positive) constants $c_{1}, c_{2}$ depending only on the family, i.e., depending only on $N$ and $d$, so that

(4) $\left|\hat{h}_{\mathcal{V}_{t}, \eta_{t}, \varphi_{t}}(x)-h_{\mathcal{V}, \eta}(x)\right| \leq c_{1} h_{T}(t)+c_{2}$

for all $t \in T^{0}(\overline{\mathbb{Q}})$ and all $x \in \mathcal{V}_{t}(\overline{\mathbb{Q}})$.

Note that for each choice of $t \in T^{0}(\overline{\mathbb{Q}})$, we get a degree $d$ morphism $\varphi_{t}: \mathbb{P}^{N} \rightarrow$ $\mathbb{P}^{N}$, and that $\hat{h}_{\mathcal{V}_{t}, \eta_{t}, \varphi_{t}}$ is then our height function $\hat{h}_{\varphi_{t}}$. Further, $h_{\mathcal{V}, \eta}$ restricted to any particular fiber $\mathcal{V}_{t}=\mathbb{P}^{N}$ is a Weil height function on $\mathbb{P}^{N}$, and $h_{T}(t)$ is simply the height $h\left(\varphi_{t}\right)$ of the morphism $\varphi_{t}$. Thus (4) becomes

$$
\left|\hat{h}_{\varphi_{t}}(x)-h(x)\right| \leq c_{1} h\left(\varphi_{t}\right)+c_{2} \quad \text { for all } x \in \mathbb{P}^{N}(\overline{\mathbb{Q}}) .
$$

Taking the supremum over $x \in \mathbb{P}^{N}(\overline{\mathbb{Q}})$ yields

$$
\hat{\delta}\left(\varphi_{t}\right) \leq c_{1} h\left(\varphi_{t}\right)+c_{2}
$$

and then Proposition 6 gives

$$
\hat{\Delta}\left(\varphi_{t}\right) \leq c_{1}^{\prime} h\left(\varphi_{t}\right)+c_{2}^{\prime}
$$

with $c_{i}^{\prime}=\left(1+d^{-1}\right) c_{i}$. This inequality holds for all $t \in T^{0}(\overline{\mathbb{Q}})$ with constants $c_{1}^{\prime}$ and $c_{2}^{\prime}$ that are independent of $t$. By construction, as $t$ varies over $T^{0}(\overline{\mathbb{Q}})$, the map $\varphi_{t}$ varies over all degree $d$ morphisms $\mathbb{P}^{N} \rightarrow \mathbb{P}^{N}$. This concludes the proof of the lower bound in (3). 
The idea underlying the proof of the upper bound in Theorem 8 is that a rational map $\varphi: \mathbb{P}^{N} \rightarrow \mathbb{P}^{N}$ is uniquely determined by its values at a sufficient number of generic points $P_{1}, \ldots, P_{K}$. More precisely, the coefficients of the polynomials defining $\varphi$ are themselves polynomial functions of the coordinates of $\varphi\left(P_{1}\right), \ldots, \varphi\left(P_{K}\right)$. In order to obtain an explicit upper bound in Theorem 8 , we determine exactly the degrees of these polynomial functions, which will enable us to prove the following key estimate.

Proposition 11. Fix integers $N \geq 1$ and $d \geq 1$, and let $K=\left(\begin{array}{c}N+d \\ d\end{array}\right)$. There a constant $C_{N, d}$ and a Zariski closed set $Z_{N, d} \subset\left(\mathbb{P}^{N}\right)^{K}$ so that for all morphisms $\varphi$ : $\mathbb{P}^{N} \rightarrow \mathbb{P}^{N}$ of degree d defined over $\overline{\mathbb{Q}}$,

$$
\begin{aligned}
& h(\varphi) \leq d(K-1) \sum_{j=1}^{K} h\left(P_{j}\right)+\sum_{j=1}^{K} h\left(\varphi\left(P_{j}\right)\right)+C_{N, d} \\
& \qquad \text { for all }\left(P_{1}, \ldots, P_{K}\right) \in\left(\mathbb{P}^{N}(\overline{\mathbb{Q}})\right)^{K} \backslash Z_{N, d} .
\end{aligned}
$$

Proof of Proposition 11. We start by setting some notation.

$\mathcal{I}_{N, d}$ The set of $(N+1)$-tuples of nonnegative integers $\left(i_{0}, \ldots, i_{N}\right)$ satisfying $i_{0}+\cdots+i_{N}=d$.

$\mathbf{X}^{I} \quad=M_{I}(\mathbf{X})=X_{0}^{i_{0}} X_{1}^{i_{1}} \cdots X_{N}^{i_{N}}$, the monomial corresponding to the $(N+1)$-tuple $I=\left(i_{0}, \ldots, i_{N}\right)$.

$K=\left(\begin{array}{c}N+d \\ N\end{array}\right)=\# \mathcal{I}_{N, d}$, the number of monomials of degree $d$ in $N+$ 1 variables.

Lemma 12. Let $\mathbf{X}^{(1)}, \mathbf{X}^{(2)}, \ldots, \mathbf{X}^{(K)}$ be $(N+1)$-tuples whose $(N+1) K$ coordinates are algebraically independent variables. Then the matrix

$$
\left(M_{I}\left(\mathbf{X}^{(j)}\right)\right)_{\substack{I \in \mathcal{I}_{N, d} \\ 1 \leq j \leq K}}
$$

whose rows are the degree $d$ monomials in the coordinates of the $\mathbf{X}^{(j)}$ has nonzero determinant. It is multihomogeneous of degree $d$ in each of $\mathbf{X}^{(1)}, \ldots, \mathbf{X}^{(K)}$.

Proof. The determinant is a sum of terms of the form

$$
\pm M_{I_{1}}\left(\mathbf{X}^{(1)}\right) \cdot M_{I_{2}}\left(\mathbf{X}^{(2)}\right) \cdots M_{I_{K}}\left(\mathbf{X}^{(K)}\right) \quad \text { with } I_{1}, \ldots, I_{K} \in \mathcal{I}_{N, d} .
$$

Each of these terms is a distinct monomial in the polynomial ring

$$
\mathbb{Z}\left[X_{0}^{(1)}, X_{1}^{(1)}, \ldots, X_{N}^{(1)}, X_{0}^{(2)}, X_{1}^{(2)}, \ldots, X_{N}^{(2)}, \ldots, X_{0}^{(K)}, X_{1}^{(K)}, \ldots, X_{N}^{(K)}\right] .
$$


Hence there can be no cancellation, so the determinant is nonzero (and in fact is a sum/difference of $K$ distinct monomials). Finally, the multihomogeneity is obvious, since each $M_{I}\left(\mathbf{X}^{(j)}\right)$ is homogeneous of degree $d$ in the coefficients of $\mathbf{X}^{(j)}$.

Resuming the proof of Proposition 11, we let

$$
\varphi=\left[\varphi_{0}: \cdots: \varphi_{N}\right]: \mathbb{P}^{N} \longrightarrow \mathbb{P}^{N}
$$

be a morphism of degree $d$, so each $\varphi_{i}(\mathbf{X})$ is a homogeneous polynomial of degree $d$. We write

$$
\varphi_{i}(\mathbf{X})=\sum_{I \in \mathcal{I}_{N, d}} a_{i I} M_{I}(\mathbf{X})
$$

so the map $\varphi$ corresponds to the point

$$
\left[a_{i I}\right]_{\substack{I \in \mathcal{I}_{N, d} \\ 0 \leq i \leq N}} \in \operatorname{Mor}_{d}^{N} \subset \mathbb{P}^{(N+1) K-1} .
$$

Let $\mathbf{X}^{(1)}, \ldots, \mathbf{X}^{(K)}$ be independent $(N+1)$-tuples as in Lemma 12 and consider the system of equations

$$
\sum_{I \in \mathcal{I}_{N, d}} a_{i I} M_{I}\left(\mathbf{X}^{(j)}\right)=\varphi_{i}\left(\mathbf{X}^{(j)}\right), \quad 1 \leq j \leq K
$$

We treat the $\mathbf{X}^{(j)}$ as fixed quantities and solve for the $a_{i I}$ coefficients. To make this precise, let $A=\left(M_{I}\left(\mathbf{X}^{(j)}\right)\right)$ be the matrix (5) defined in Lemma 12, let $B=$ $A^{\text {adj }}$ be the adjoint matrix, and let $D=\operatorname{det}(A)$. Then we obtain

$$
D a_{i I}=\sum_{1 \leq j \leq K} B_{j I} \varphi_{i}\left(\mathbf{X}^{(j)}\right), \quad I \in \mathcal{I}_{N, d}
$$

The coordinates of the $j^{\text {th }}$ row of the matrix $A$ are the degree $d$ monomials in the coordinates of $\mathbf{X}^{(j)}$. The coordinates of the adjoint matrix $B=A^{\text {adj }}$ are sums/differences of terms, each of which is a product of $K-1$ entries from $A$. More precisely, the entry $B_{j I}$ is a sum/difference of monomials, each of which is multihomogenous of degree $d$ in the $K-1$ variables

$$
\mathbf{X}^{(1)}, \mathbf{X}^{(2)}, \ldots, \mathbf{X}^{(j-1)}, \mathbf{X}^{(j+1)}, \ldots, \mathbf{X}^{(K)} .
$$

For convenience we write $B_{j I}\left(\mathbf{X}^{(1)}, \ldots, \mathbf{X}^{(K)}\right)$, although in fact $B_{j I}$ does not depend on $\mathbf{X}^{(j)}$. 
We now define a rational map

$$
\begin{gathered}
U:\left(\mathbb{P}^{N} \times \mathbb{P}^{N}\right)^{K} \longrightarrow \mathbb{P}^{(N+1) K-1} \\
U\left(P_{1}, Q_{1}, \ldots, P_{K}, Q_{K}\right)=\left[\sum_{1 \leq j \leq K} B_{j I}\left(P_{1}, \ldots, P_{K}\right) X_{i}\left(Q_{j}\right)\right]_{\substack{I \in \mathcal{I}_{N, d} \\
0 \leq i \leq N}}
\end{gathered}
$$

where $X_{i}(Q)$ denotes the $i^{\text {th }}$ coordinate of $Q$. Notice that $U$ is multihomogeneous of degree $d(K-1)$ in the variables $P_{1}, \ldots, P_{K}$ and it is multihomogeneous of degree 1 in the variables $Q_{1}, \ldots, Q_{K}$.

From the way that we have set up these equations, we have for all $P_{1}, \ldots, P_{K} \in$ $\mathbb{P}^{N}$

$$
U\left(P_{1}, \varphi\left(P_{1}\right), P_{2}, \varphi\left(P_{2}\right), \ldots, P_{K}, \varphi\left(P_{K}\right)\right)=\left[D\left(P_{1}, \ldots, P_{K}\right) a_{i I}\right]_{\substack{I \in \mathcal{I}_{N, d} \\ 0 \leq i \leq N}} .
$$

Hence if $P_{1}, \ldots, P_{K} \in \mathbb{P}^{N}$ satisfy $D\left(P_{1}, \ldots, P_{N}\right) \neq 0$, then

$$
\begin{aligned}
U\left(P_{1}, \varphi\left(P_{1}\right), P_{2}, \varphi\left(P_{2}\right), \ldots,\right. & \left.P_{K}, \varphi\left(P_{K}\right)\right) \\
& =\left[a_{i I}\right]=[\varphi] \in \operatorname{Mor}_{d}^{N} \subset \mathbb{P}^{(N+1) K-1} .
\end{aligned}
$$

Taking heights and using the multihomogeneity of $U$, we obtain

$$
\begin{aligned}
h(\varphi) & =h\left(U\left(P_{1}, \varphi\left(P_{1}\right) \ldots, P_{K}, \varphi\left(P_{K}\right)\right)\right) \\
& \leq d(K-1) \sum_{j=1}^{K} h\left(P_{j}\right)+\sum_{j=1}^{K} h\left(\varphi\left(P_{j}\right)\right)+O_{N, d}(1) .
\end{aligned}
$$

Note that the inequality in this direction is a simple consequence of the triangle inequality, we do not need $U$ to be a morphism. (Indeed, $U$ is not a morphism.) And it would not be hard to obtain an explicit value for the $O_{N, d}(1)$ constant, although we shall not do so.

In order to complete the proof of the upper bound in Theorem 8 we exploit the fact that the points of height zero are Zariski dense, as described in the following lemma.

Lemma 13. Let $V_{1}, \ldots, V_{N}$ be projective varieties, and for each $i$, let $T_{i} \subset V_{i}$ be a Zariski dense set of points. Then the product $T=T_{1} \times \cdots \times T_{N}$ is Zariski dense in $V=V_{1} \times \cdots \times V_{N}$.

In particular,

$$
\left\{P \in \mathbb{P}^{N}(\overline{\mathbb{Q}}): h(P)=0\right\}
$$


is Zariski dense in $\mathbb{P}^{N}$.

Proof. The proof is by induction on $N$. For $N=1$ the assertion is clear. Assume it is true for $N-1$. Let $f$ be a rational function on $V$ that vanishes on $T$ and let $t_{1} \in T_{1}$. (We may assume that the support of the polar divisor of $f$ does not contain the set $\left\{t_{1}\right\} \times V_{2} \times \cdots \times V_{N}$.) Then by assumption, the rational function

$$
g_{t_{1}}\left(x_{2}, \ldots, x_{N}\right)=f\left(t_{1}, x_{2}, \ldots, x_{N}\right) \quad \text { on } V_{2} \times \cdots \times V_{N}
$$

vanishes on $T_{2} \times \cdots \times T_{N}$. By induction, the set $T_{2} \times \cdots \times T_{N}$ is Zariski dense in $V_{2} \times \cdots \times V_{N}$, so we conclude that $g_{t_{1}}$ is identically 0 on $V_{2} \times \cdots \times V_{N}$. Hence for any choice of points $\left(y_{2}, \ldots, y_{N}\right) \in V_{2} \times \cdots \times V_{N}$, the rational function

$$
f\left(x_{1}, y_{2}, \ldots, y_{N}\right) \text { on } V_{1}
$$

vanishes on $T_{1}$. Since $T_{1}$ is Zariski dense in $V_{1}$, it follows that if vanishes for all $x_{1} \in V_{1}$. This proves that $f$ is identically 0 on $V$. Hence $T$ is Zariski dense in $V$.

For the second statement, we observe that $h(P)=0$ for those points all of whose coordinates are either 0 or roots of unity. Let $\boldsymbol{\mu}_{\infty} \subset \overline{\mathbb{Q}}^{*} \subset \mathbb{P}^{1}(\overline{\mathbb{Q}})$ denote the set of all roots of unity. Then $\boldsymbol{\mu}_{\infty}$ is infinite, so it is dense in $\mathbb{P}^{1}$, and hence $\boldsymbol{\mu}_{\infty}^{N}$ is Zariski dense in $\left(\mathbb{P}^{1}\right)^{N}$. But $\left(\mathbb{P}^{1}\right)^{N}$ is birational to $\mathbb{P}^{N}$, so

$$
\left\{\left[1: \zeta_{1}: \cdots: \zeta_{N}\right]: \zeta_{1}, \ldots, \zeta_{N} \in \boldsymbol{\mu}_{\infty}\right\}
$$

is Zariski dense in $\mathbb{P}^{N}$.

Proof of the Upper Bound in Theorem 8. Let $C_{N, d}, Z_{N, d}$, and $K$ be as in the statement of Proposition 11. From the second part of Lemma 13, we know that the points of Weil height 0 are dense in $\mathbb{P}^{N}(\overline{\mathbb{Q}})$, and then the first part of Lemma 13 tells us that

$$
\left\{\left(P_{1}, \ldots, P_{K}\right) \in \mathbb{P}^{N}(\overline{\mathbb{Q}})^{K}: h\left(P_{1}\right)=\cdots=h\left(P_{K}\right)=0\right\}
$$

is Zariski dense in $\left(\mathbb{P}^{N}\right)^{K}$. In particular, we can find a $K$-tuple of points $\left(P_{1}, \ldots, P_{K}\right)$ in the set (6) that is not in the Zariski closed set $Z_{N, d}$. Then Proposition 11 gives the estimate

$$
h(\varphi) \leq \sum_{j=1}^{K} h\left(\varphi\left(P_{j}\right)\right)+C_{N, d} .
$$


Using the fact that every $h\left(P_{j}\right)=0$, we rewrite this as

$$
\begin{aligned}
h(\varphi) & \leq d K \max _{1 \leq j \leq K}\left(\frac{1}{d} h\left(\varphi\left(P_{j}\right)\right)-h\left(P_{j}\right)\right)+C_{N, d} \\
& \leq d K \sup _{P \in \mathbb{P}^{N}(\overline{\mathbb{Q}})}\left|\frac{1}{d} h(\varphi(P))-h(P)\right|+C_{N, d} \\
& =d K \hat{\Delta}(\varphi)+C_{N, d} .
\end{aligned}
$$

This completes the proof of the upper bound in Theorem 8 .

Remark 14. Our elementary proof of Proposition 11 is via a direct matrix calculation. Zhang [14, Theorem 5.2] has proven that if $V \subset \mathbb{P}^{N}$ is a variety defined over $\overline{\mathbb{Q}}$, then

$$
h(V) \leq \sup _{Z \subsetneq V} \inf _{P \in(V \backslash Z)(\overline{\mathbb{Q}})} h(P),
$$

where the supremum is over Zariski closed subsets of $V$ and where the height $h(V)$ of the variety $V$ is defined using arithmetic intersection theory and the FubiniStudy metric on $\mathbb{P}^{N}$. (See $[1,2,14]$ for further details.) Applying Zhang's inequality to various projections, we can prove a version of Theorem 8 of the form

$$
h\left(\varphi_{i}\right) \leq N d \hat{\Delta}(\varphi)+O_{N, d}(1) \quad \text { for } i=0,1, \ldots, N .
$$

This is somewhat weaker than the upper bound (3) in Theorem 8 , since it involves the individual coordinate functions of $\varphi$, but the constant is better. It would be interesting to try to use Zhang's inequality directly to prove Proposition 11 and Theorem 8. One possibility might be to apply Zhang's result to the graph

$$
V=\left\{(P, \varphi(P)): P \in \mathbb{P}^{N}\right\} \subset \mathbb{P}^{N} \times \mathbb{P}^{N},
$$

thereby obtaining an estimate that simultaneously involves all of the coordinate functions of $\varphi$, but we will not pursue this idea further in this paper.

\section{Finiteness Properties}

We combine the various comparison results to prove Theorem 1, which we restate here for the convenience of the reader.

Theorem 15. Let $\varphi, \psi: \mathbb{P}^{N} \rightarrow \mathbb{P}^{N}$ be morphisms of degree at least 2 defined over $\overline{\mathbb{Q}}$. Then

$$
\hat{\delta}(\varphi, \psi)-h(\psi) \ll h(\varphi) \ll \hat{\delta}(\varphi, \psi)+h(\psi)
$$


where the implied constants depend only on $N$ and the degrees of $\varphi$ and $\psi$. For the upper bound, letting $d=\operatorname{deg}(\varphi)$, we obtain an explicit estimate of the form

$$
h(\varphi) \leq(d+1)\left(\begin{array}{c}
N+d \\
N
\end{array}\right) \hat{\delta}(\varphi, \psi)+O_{N, d}(h(\psi)) .
$$

Proof. For convenience, let $\lambda$ be the squaring map, so $\hat{h}_{\lambda}$ is the usual Weil height $h$. Also let $K=\left(\begin{array}{c}N+d \\ N\end{array}\right)$ as usual. We estimate

$$
\begin{aligned}
h(\varphi) & \leq K d \hat{\Delta}_{\lambda}(\varphi)+O(1) & & \text { from Theorem 8, } \\
& \leq K(d+1) \hat{\delta}(\varphi, \lambda)+O(1) & & \text { from Prop. 6, } \\
& \leq K(d+1)(\hat{\delta}(\varphi, \psi)+\hat{\delta}(\psi, \lambda))+O(1) & & \text { from Lemma 5, } \\
& \leq K(d+1) \hat{\delta}(\varphi, \psi)+2 K(d+1) \hat{\Delta}_{\lambda}(\psi)+O(1) & & \text { from Prop. 6, } \\
& \leq K(d+1) \hat{\delta}(\varphi, \psi)+O(h(\psi)) & & \text { from Theorem 8. }
\end{aligned}
$$

This gives the upper bound. The lower bound is proven similarly, we leave the details to the reader.

Definition. Let $\varphi: \mathbb{P}^{N} \rightarrow \mathbb{P}^{N}$ be a morphism defined over $\overline{\mathbb{Q}}$. The field of definition of $\varphi$, denoted $\mathbb{Q}(\varphi)$, is the fixed field of

$$
\left\{\sigma \in \operatorname{Gal}(\overline{\mathbb{Q}} / \mathbb{Q}): \varphi^{\sigma}=\varphi\right\} .
$$

Equivalently, $\mathbb{Q}(\varphi)$ is the field generated by the coordinates of the point in $\operatorname{Mor}_{d}^{N}(\overline{\mathbb{Q}}) \subset \mathbb{P}^{L}(\overline{\mathbb{Q}})$ associated to $\varphi$.

Corollary 16. Fix a morphism $\psi: \mathbb{P}^{N} \rightarrow \mathbb{P}^{N}$ of degree at least 2 defined over $\overline{\mathbb{Q}}$ and an integer $d \geq 2$. Then for all $B>0$ the set of morphisms $\varphi \in \operatorname{Mor}_{d}^{N}(\overline{\mathbb{Q}})$ satisfying

$$
\hat{\delta}(\varphi, \psi) \leq B
$$

is a set of bounded height in $\operatorname{Mor}_{d}^{N}(\overline{\mathbb{Q}}) \subset \mathbb{P}^{L}(\overline{\mathbb{Q}})$.

In particular, with $\psi$ fixed as above and for any constants $B, C, D$, there are only finitely many morphisms $\varphi: \mathbb{P}^{N} \rightarrow \mathbb{P}^{N}$ defined over $\overline{\mathbb{Q}}$ and satisfying

$$
2 \leq \operatorname{deg}(\varphi) \leq D, \quad[\mathbb{Q}(\varphi): \mathbb{Q}] \leq C, \quad \text { and } \quad \hat{\delta}(\varphi, \psi) \leq B
$$

Proof. Using Theorem 15, the assumption that $\hat{\delta}(\varphi, \psi) \leq B$ implies that $h(\varphi) \ll$ $B+h(\psi)$ is bounded, which proves the first assertion. Then the second statement follows immediately from Northcott's theorem, which says that there are only 
finitely many points of bounded height and degree in projective space (see $[4$, B.2.3] or [7, Chapter 3, Theorem 2.6]).

\section{Dynamics AND A PGL-InVARIANt ARIthmetic Distance}

The dynamical properties of a morphism $\varphi: \mathbb{P}^{N} \rightarrow \mathbb{P}^{N}$ are essentially unchanged if $\varphi$ is replaced by a PGL-conjugate

$$
\varphi^{f}(P)=\left(f^{-1} \circ \varphi \circ f\right)(P) \quad \text { for some } f \in \operatorname{Aut}\left(\mathbb{P}^{N}\right)=\mathrm{PGL}_{N+1} .
$$

This naturally leads one to study the quotient space

$$
M_{d}^{N}=\operatorname{Mor}_{d}^{N} / \mathrm{PGL}_{N+1} .
$$

In particular, Milnor constructed $M_{d}^{1}(\mathbb{C})$ as a complex orbifold [9] and the second author used geometric invariant theory to construct $M_{d}^{1}$ as a variety over $\mathbb{Q}$ (and as a scheme over $\mathbb{Z}$ ), see [12]. We expect more generally that $M_{d}^{N}$ has the structure of a variety over $\mathbb{Q}$ (and a scheme over $\mathbb{Z}$ ), although this result does not seem to have yet appeared in the literature.

In any case, it is natural to define arithmetic distances and arithmetic complexity for PGL-equivalence classes of morphisms. For convenience we write $[\varphi] \in M_{d}^{N}$ for the PGL-equivalence class containing the morphism $\varphi$.

Definition. Let $\varphi, \psi: \mathbb{P}^{N} \rightarrow \mathbb{P}^{N}$ be morphisms of degree at least 2 defined over $\overline{\mathbb{Q}}$. The (dynamical) arithmetic distance from $[\varphi]$ to $[\psi]$ is

$$
\hat{\delta}([\varphi],[\psi])=\inf _{f, g \in \mathrm{PGL}_{N+1}(\overline{\mathbb{Q}})} \hat{\delta}\left(\varphi^{f}, \psi^{g}\right) .
$$

We note some elementary properties of canonical heights and arithmetic distances under PGL-conjugation.

Proposition 17. Let $\varphi, \psi: \mathbb{P}^{N} \rightarrow \mathbb{P}^{N}$ be morphisms of degree at least 2 defined over $\overline{\mathbb{Q}}$ and let $f, g \in \mathrm{PGL}_{N+1}(\overline{\mathbb{Q}})$.

(a) $\hat{h}_{\varphi^{f}}(P)=\hat{h}_{\varphi}(f(P))$.

(b) $\hat{\delta}\left(\varphi^{f}, \psi^{g}\right)=\hat{\delta}\left(\varphi^{f g^{-1}}, \psi\right)$.

(c) $\hat{\delta}([\varphi],[\psi])=\inf _{f \in \mathrm{PGL}_{N+1}(\overline{\mathbb{Q}})} \sup _{P \in \mathbb{P}^{N}(\overline{\mathbb{Q}})}\left|\hat{h}_{\varphi}(f(P))-\hat{h}_{\psi}(P)\right|$. 
Proof. (a) Let $d=\operatorname{deg}(\varphi)$. Then

$$
\begin{aligned}
\hat{h}_{\varphi^{f}}(P) & =\lim _{n \rightarrow \infty} \frac{1}{d^{n}} h\left(\left(\varphi^{f}\right)^{n}(P)\right) \\
& =\lim _{n \rightarrow \infty} \frac{1}{d^{n}} h\left(\left(f^{-1} \circ \varphi^{n} \circ f\right)(P)\right) \\
& =\lim _{n \rightarrow \infty} \frac{1}{d^{n}}\left(h\left(\left(\varphi^{n} \circ f\right)(P)\right)+O_{f}(1)\right) \\
& =\hat{h}_{\varphi}(f(P)) .
\end{aligned}
$$

(b) We compute

$$
\begin{aligned}
\hat{\delta}\left(\varphi^{f}, \psi^{g}\right) & =\sup _{P \in \mathbb{P}^{N}(\overline{\mathbb{Q}})}\left|\hat{h}_{\varphi^{f}}(P)-\hat{h}_{\psi^{g}}(P)\right| & \text { definition of } \hat{\delta} \\
& =\sup _{P \in \mathbb{P}^{N}(\overline{\mathbb{Q}})}\left|\hat{h}_{\varphi^{f}}\left(g^{-1}(P)\right)-\hat{h}_{\psi^{g}}\left(g^{-1}(P)\right)\right| & \\
& \left.=\sup _{P \in \mathbb{P}^{N}(\overline{\mathbb{Q}})} \mid \hat{h}_{\varphi^{f g^{-1}}}(P)-\hat{h}_{\psi}(P)\right) \mid & \\
& =\hat{\delta}\left(\varphi^{f g^{-1}}, \psi\right) . & \text { from (a), }
\end{aligned}
$$

(c) We compute

$$
\begin{aligned}
\hat{\delta}([\varphi],[\psi]) & =\inf _{f, g \in \mathrm{PGL}_{N+1}(\overline{\mathbb{Q}})} \hat{\delta}\left(\varphi^{f}, \psi^{g}\right) & & \text { definition of } \hat{\delta}, \\
& =\inf _{f, g \in \mathrm{PGL}_{N+1}(\overline{\mathbb{Q}})} \hat{\delta}\left(\varphi^{f g^{-1}}, \psi\right) & & \text { from (b), } \\
& =\inf _{f \in \operatorname{PGL}_{N+1}(\overline{\mathbb{Q}})} \hat{\delta}\left(\varphi^{f}, \psi\right) & & \\
& =\inf _{f \in \operatorname{PGL}_{N+1}(\overline{\mathbb{Q}})} \sup _{P \in \mathbb{P}^{N}(\overline{\mathbb{Q}})}\left|\hat{h}_{\varphi f}(P)-\hat{h}_{\psi}(P)\right| & & \text { definition of } \hat{\delta}, \\
& =\inf _{f \in \operatorname{PGL}_{N+1}(\overline{\mathbb{Q}})} \sup _{P \in \mathbb{P}^{N}(\overline{\mathbb{Q}})}\left|\hat{h}_{\varphi}(f(P))-\hat{h}_{\psi}(P)\right| & & \text { from (a). }
\end{aligned}
$$

We conclude by asking if there is a single $f \in \mathrm{PGL}_{N+1}(\overline{\mathbb{Q}})$ that achieves the infimum in the definition of arithmetic distance on $M_{d}^{N}$.

Question 18. Let $\varphi, \psi: \mathbb{P}^{N} \rightarrow \mathbb{P}^{N}$ be morphisms of degree at least 2 defined over $\overline{\mathbb{Q}}$. Does there always exist an $f \in \mathrm{PGL}_{N+1}(\overline{\mathbb{Q}})$ such that

$$
\hat{\delta}([\varphi],[\psi])=\hat{\delta}\left(\varphi^{f}, \psi\right) ?
$$




\section{REFERENCES}

[1] Ahmed Abbes. Hauteurs et discrétude (d'après L. Szpiro, E. Ullmo et S. Zhang). Astérisque, (245):Exp. No. 825, 4, 141-166, 1997. Séminaire Bourbaki, Vol. 1996/97.

[2] J.-B. Bost, H. Gillet, and C. Soulé. Heights of projective varieties and positive Green forms. J. Amer. Math. Soc., 7(4):903-1027, 1994.

[3] Gregory S. Call and Joseph H. Silverman. Canonical heights on varieties with morphisms. Compositio Math., 89(2):163-205, 1993.

[4] Marc Hindry and Joseph H. Silverman. Diophantine geometry, volume 201 of Graduate Texts in Mathematics. Springer-Verlag, New York, 2000. An introduction.

[5] Shu Kawaguchi. Canonical heights for random iterations in certain varieties. Int. Math. Res. Notices, no. 7 article ID rnm023, 33 pages, 2007.

[6] Shu Kawaguchi and Joseph H. Silverman. Dynamics of projective morphisms having identical canonical heights. Proc. London Math. Soc., 95(3):519-544, 2007. Addendum, 97(1): 272,2008

[7] Serge Lang. Fundamentals of Diophantine geometry. Springer-Verlag, New York, 1983.

[8] Ju. I. Manin. The Tate height of points on an Abelian variety, its variants and applications. Izv. Akad. Nauk SSSR Ser. Mat., 28:1363-1390, 1964.

[9] John Milnor. Geometry and dynamics of quadratic rational maps. Experiment. Math., 2(1):37-83, 1993. With an appendix by the author and Lei Tan.

[10] A. Néron. Quasi-fonctions et hauteurs sur les variétés abéliennes. Ann. of Math. (2), 82:249$331,1965$.

[11] Joseph H. Silverman. Computing heights on elliptic curves. Math. Comp., 51(183):339-358, 1988.

[12] Joseph H. Silverman. The space of rational maps on $\mathbf{P}^{1}$. Duke Math. J., 94(1):41-77, 1998.

[13] Heinz M. Tschöpe and Horst G. Zimmer. Computation of the Néron-Tate height on elliptic curves. Math. Comp., 48(177):351-370, 1987.

[14] Shouwu Zhang. Positive line bundles on arithmetic varieties. J. Amer. Math. Soc., 8(1):187$221,1995$.

Shu Kawaguchi

Department of Mathematics, Graduate School of Science

Osaka University, Toyonaka, Osaka 560-0043 Japan

E-mail: kawaguch@math.sci.osaka-u.ac.jp

Joseph H. Silverman

Mathematics Department, Box 1917 Brown University

Providence, RI 02912 USA

E-mail: jhs@math.brown.edu 